\title{
GABA Is Depolarizing in Hippocampal Dentate Granule Cells of the Adolescent and Adult Rats
}

\author{
Po-Han Chiang, ${ }^{1 \star} \mathrm{Pu}-Y e h \mathrm{Wu},{ }^{1 \star}$ Tzu-Wei Kuo, ${ }^{1 \star}$ Yu-Chao Liu, ${ }^{1}$ Chu-Fang Chan, ${ }^{1}$ Ta-Chun Chien, ${ }^{2,3}$ Jen-Kun Cheng, ${ }^{4}$ \\ Yu-Yin Huang, ${ }^{5}$ Cheng-Di Chiu, ${ }^{6}$ and Cheng-Chang Lien ${ }^{1}$ \\ ${ }^{1}$ Institute of Neuroscience and Brain Research Center and ${ }^{2}$ Institute of Biochemistry and Molecular Biology, School of Life Sciences, National Yang-Ming \\ University, Taipei 112, Taiwan, ${ }^{3}$ Molecular Medicine Program, Taiwan International Graduate Program, Institute of Biomedical Sciences, Academia Sinica, \\ Taipei 115, Taiwan, ${ }^{4}$ Department of Anesthesiology, Mackay Memorial Hospital, Taipei 104, Taiwan, Mackay Medicine, Nursing and Management College, \\ Taipei 112, Taiwan, Department of Anesthesiology, Taipei Medical University, Taipei 110, Taiwan, ${ }^{5}$ Department of Anesthesiology, Cheng Hsin General \\ Hospital, Taipei 112, Taiwan, and 'Department of Neurosurgery, China Medical University Hospital, Taichung 404, Taiwan
}

GABAergic signaling in hippocampal pyramidal neurons undergoes a switch from depolarizing to hyperpolarizing during early neuronal development. Whether such a transformation of GABAergic action occurs in dentate granule cells (DGCs), located at the first stage of the hippocampal trisynaptic circuit, is unclear. Here, we use noninvasive extracellular recording to monitor the effect of synaptically released GABA on the DGC population. We find that GABAergic responses in adolescent and adult rat DGCs are still depolarizing from rest. Using a morphologically realistic DGC model, we show that GABAergic action, depending on its precise timing and location, can have either an excitatory or inhibitory role in signal processing in the dentate gyrus.

\section{Introduction}

GABA, the main inhibitory neurotransmitter in the adult brain, exerts its fast action through activation of anion (predominantly $\mathrm{Cl}^{-}$)-permeant $\mathrm{GABA}_{\mathrm{A}}$ receptors (Bartos et al., 2007). Early in development, however, the action of GABA can be both depolarizing and excitatory (Rivera et al., 1999; for review, see Ben-Ari, 2002; Rivera et al., 2005). The switch from an excitatory to inhibitory action of $\mathrm{GABA}_{\mathrm{A}}$ receptors is attributable to a developmental shift in the intracellular chloride concentration, $\left[\mathrm{Cl}^{-}\right]_{\mathrm{i}}$. In hippocampal pyramidal neurons $(\mathrm{PNs}),\left[\mathrm{Cl}^{-}\right]_{\mathrm{i}}$ is regulated by the differential expression of the $\mathrm{Na}^{+}-\mathrm{K}^{+}-2 \mathrm{Cl}^{-}$cotransporter 1 (NKCC1, a $\mathrm{Cl}^{-}$importer) and the $\mathrm{K}^{+}{ }_{-} \mathrm{Cl}^{-}$cotransporter 2 (KCC2, a Cl${ }^{-}$exporter) (Rivera et al., 1999; Ben-Ari, 2002). In immature PNs, NKCC1 expression is high, whereas KCC2 expression is low, leading to high $\left[\mathrm{Cl}^{-}\right]_{\mathrm{i}}$. During neuronal maturation, KCC2 expression is steeply upregulated. As a result, in the adult hippocampal PNs KCC2 maintains a low $\left[\mathrm{Cl}^{-}\right]_{\mathrm{i}}$, such that $\mathrm{GABA}_{\mathrm{A}}$ receptor activation leads to $\mathrm{Cl}^{-}$influx and hyperpolar-

\footnotetext{
Received July 4, 2011; revised 0ct. 26, 2011; accepted Nov. 4, 2011

Author contributions: J.-K.C., Y.-Y.H., C.-D.C., and C.-C.L. designed research; P.-H.C., P.-Y.W., T.-W.K., Y.-C.L., C.-F.C., and T.-C.C. performed research; P.-H.C., P.-Y.W., T.-W.K., Y.-C.L., C.-F.C., T.-C.C., and C.-C.L. analyzed data; C.-C.L. wrote the paper.

This work was supported by grants from the Ministry of Education, Aim for the Top University Plan, National Health Research Institutes (NHRI-EX100-9720NC), Taiwan National Science Council (NSC99-2321-B-010-001), and Cheng Hsin General Hospital (Grant Nr. 100-53 to Y.-Y.H). We thank Drs. G. J. Stuart and C. Schmidt-Hieber for critically reading the manuscript, P. Jonas for comments on the initial experiments, and Drs. G. J. Stuart, C. SchmidtHieber, M. Martina, and R. Piet for helpful advice.

*P.-H.C., P.-Y.W., and T.-W.K. contributed equally to this work.

Correspondence should be addressed to Dr. Cheng-Chang Lien, Institute of Neuroscience and Brain Research Center, National Yang-Ming University 155, Section 2, Li-Nong Street, Taipei 112, Taiwan. E-mail: cclien@ym.edu.tw.

DOI:10.1523/JNEUROSCI.3393-11.2012

Copyright $\odot 2012$ the authors $\quad 0270-6474 / 12 / 320062-06 \$ 15.00 / 0$
}

ization of the membrane potential (Rivera et al., 1999; Ben-Ari, 2002; Banke and McBain, 2006).

In contrast, the action of GABAergic inputs onto dentate granule cells (DGCs), a key relay station that controls information transfer from the entorhinal cortex into the hippocampus proper (Amaral et al., 2007), remains inconclusive. Recent studies showed that GABAergic action switches from "depolarizing" to "hyperpolarizing" during maturation of DGCs (Ge et al., 2006; Sun et al., 2009; Ming and Song, 2011). Yet these findings contrast with a previous report that GABAergic inputs onto DGCs are depolarizing (Staley and Mody, 1992) and are contradictory to the relatively hyperpolarized resting potential $\left(V_{\text {rest }}\right)$ of DGCs, which is negative to the reversal potential for GABAergic currents $\left(E_{\mathrm{GABA}}\right)$ (Staley and Mody, 1992; Kraushaar and Jonas, 2000).

In this study, we reexamined the fundamental question whether GABAergic interneurons depolarize or hyperpolarize their targeted DGCs in the adolescent and adult rats. By using a combination of extracellular unitary field potential (uField) recording and post hoc identification of the recorded interneuron axonal arbor, we demonstrated that both perisomatic-targeting interneurons (P-INs) and dendritic-targeting interneurons (D-INs) depolarize DGCs. Further analysis of morphologically realistic DGC cable models reveals that GABAergic action, depending on its precise timing and location, could have a biphasic effect on synaptic integration in the dentate gyrus (DG).

\section{Materials and Methods}

Slice preparation, electrophysiology, and labeling. Transverse hippocampal slices $(300 \mu \mathrm{m})$ were prepared from male adolescent [postnatal day 21 (P21)-P42] and adult (P43-P93) Sprague Dawley rats, according to methods approved by the Animal Care and Use Committee of National Yang-Ming University. Slices were sectioned in the ice-cold buffer containing the following (in mM): $87 \mathrm{NaCl}, 25 \mathrm{NaHCO}_{3}, 1.25 \mathrm{NaH}_{2} \mathrm{PO}_{4}, 2.5$ 
A

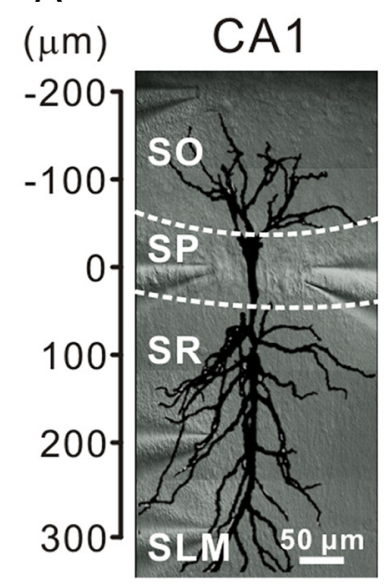

C

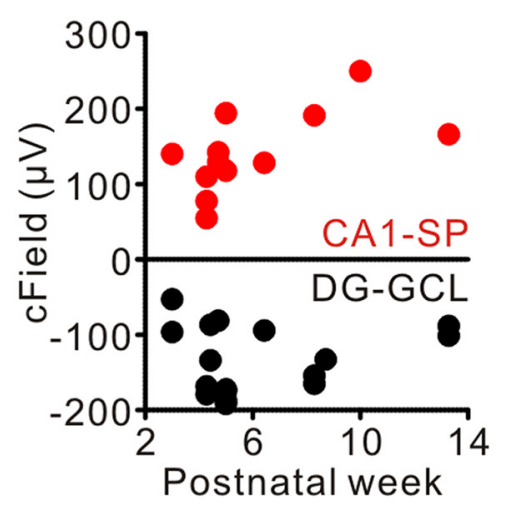

B

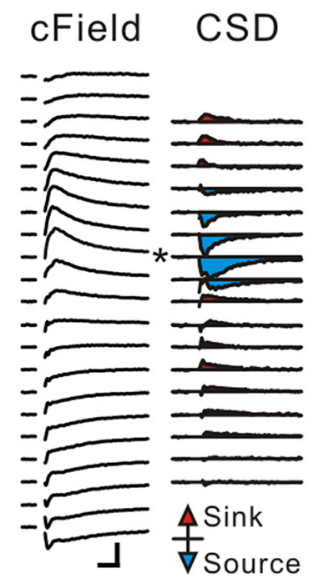

D

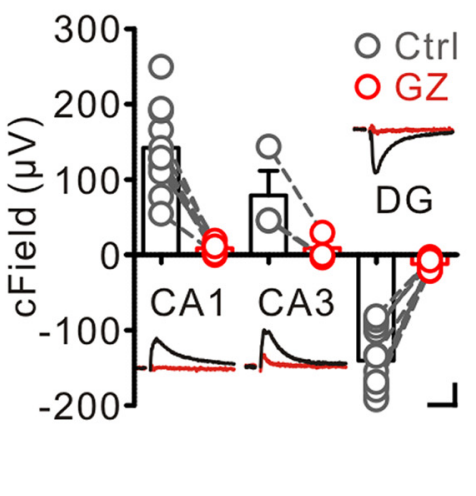

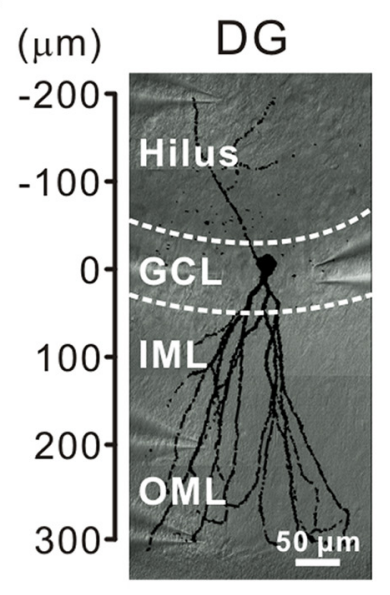

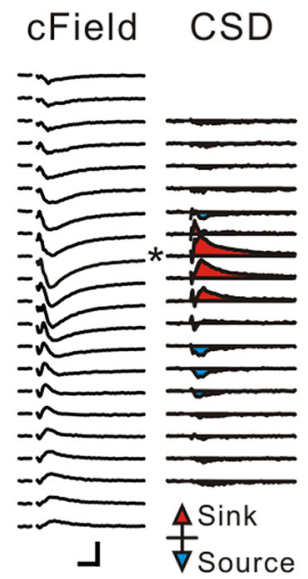

$\mathrm{E}$

Figure 1. GABAergic action in the hippocampus. A, Left, Recording configuration. Center, A laminar profile of cFields, which were sequentially recorded by an electrode (left) positioned in multiple layers of the CA1 area. The stimulation electrode (right) was placed within the SP. Calibration: $5 \mathrm{~ms}, 100 \mu$ V. Right, CSD analysis of cFields. Asterisk, The initiation site of current source signal. SO, stratum oriens; SR, stratum radiatum; SLM, stratum lacunosum-moleculare. $\boldsymbol{B}$, Recordings and analysis similar to those in $\boldsymbol{A}$; the stimulation electrode (right) was placed within the GCL. Calibration: $5 \mathrm{~ms}, 50 \mu \mathrm{V}$. Asterisk, The initiation site of current sink signal. C, Plot of cField recorded in the CA1-SP and DG-GCL areas against postnatal ages. D, Summary of cFields recorded in the CA-SP and DG-GCL areas before and after GZ treatment. Traces, Representative cFields recorded from indicated areas. Calibration: $20 \mathrm{~ms}, 100 \mu \mathrm{V}$. $\boldsymbol{E}$, Summary of $\tau_{\text {rise }}$ and $\tau_{\text {decay }}$ of cFields recorded from the CA-SP and DG-GCL areas.

$\mathrm{KCl}, 10$ glucose, 75 sucrose, $0.5 \mathrm{CaCl}_{2}$ and $7 \mathrm{MgCl}_{2}$, recovered (25 min, $\left.34^{\circ} \mathrm{C}\right)$ in the same buffer $\left(95 \% \mathrm{O}_{2} / 5 \% \mathrm{CO}_{2}\right)$, then stored at room temperature. During experiments, each slice was transferred to a submerged chamber and was superfused with oxygenated artificial CSF (ACSF) containing the following (in mM): $125 \mathrm{NaCl}, 25 \mathrm{NaHCO}_{3}, 1.25 \mathrm{NaH}_{2} \mathrm{PO}_{4}, 2.5$ $\mathrm{KCl}, 25$ glucose, $2 \mathrm{CaCl}_{2}$, and $1 \mathrm{MgCl}_{2}$.

Borosilicate glass electrodes used for Field recordings were filled with ACSF. Compound field potentials (cFields) were evoked by glass electrodes (tip diameter $\sim 20 \mu \mathrm{m}$ ) placed in different layers of CA and DG areas. Pulses (3-10 V; $100 \mu$ s) were delivered every $30 \mathrm{~s}$ using a stimulus isolator (Isoflex, A.M.P.I.). For uField recording, single action potentials (APs) were generated in interneurons by current injection in the currentclamp configuration. Interneurons were visually selected for whole-cell patch recordings (3-6 M $\Omega$ electrode resistance) under differential interference contrast optics (BX51WI, Olympus) using Multiclamp 700B or Axopatch 200B amplifiers (Molecular Devices). Data were filtered at 2 $\mathrm{kHz}$, and sampled at $10 \mathrm{kHz}$ with a Digidata 1440 interface (Molecular Devices) controlled by pClamp 10.2 software (Molecular Devices). Example traces were the average of 20-100 sweeps. Recordings were made at $22-24^{\circ} \mathrm{C}$, except uField recordings $\left(34^{\circ} \mathrm{C}\right)$.

The internal solution contained the following (in $\mathrm{mm}$ ): 135 K-gluconate, $20 \mathrm{KCl}, 0.1 \mathrm{EGTA}, 2 \mathrm{MgCl}_{2}, 4 \mathrm{Na}_{2} \mathrm{ATP}, 10$ HEPES and 0.3 $\mathrm{Na}_{3} \mathrm{GTP}$ ( $\mathrm{pH} 7.3 ; \mathrm{HCl}$ ). Gabazine (GZ) was from Tocris Bioscience; $\mathrm{N}$-cyclopentyladenosine (CPA) and 8-cyclopentyl-1,3-dipropylxanthine (DPCPX) were from Ascent Scientific. All other chemicals were from Sigma except where noted.
Neurons were filled with biocytin $(2 \mathrm{mg} / \mathrm{ml})$ during whole-cell recordings and subsequently fixed overnight with $4 \%$ paraformaldehyde in phosphate-buffered solution ( $\mathrm{PB} ; 0.1 \mathrm{M}, \mathrm{pH} 7.3$ ). After washing with $\mathrm{PB}$, slices were incubated with fluorescein isothiocyanate-conjugated avidin-d ( $2 \mu \mathrm{l} / \mathrm{ml}$; Invitrogen) in PB and $0.3 \%$ Triton X-100 overnight at $4^{\circ} \mathrm{C}$. After wash, slices were embedded in mounting medium Vectashield (Vector Laboratories). Labeled cells were imaged by a two-photon microscope as described previously (Weng et al., 2010) and reconstructed with the Neuromantic 1.6.3 software (developed by Darren Myatt, University of Reading, Berkshire, UK). The current source-density (CSD) analysis was performed as described by Mitzdorf (1985). Briefly, cFields were recorded at different locations (interval $25 \mu \mathrm{m}$ ) along a track vertical to the principal cell layer of CA areas or the granule cell layer (GCL). The one-dimensional CSD function $i_{\mathrm{m}}(t)$ was calculated from the second spatial derivative of Field $V(t, x)$, using a spatial differentiation grid $(\Delta x)$ of $50 \mu \mathrm{m}$.

$$
i_{\mathrm{m}}(t) \approx \frac{\partial^{2} V(t, x)}{\partial x^{2}}=\frac{V(t, x+\Delta x)-2 V(t, x)+V(t, x-\Delta x)}{\Delta x^{2}} .
$$

Since the CSD was calculated in one dimension and the value of the extracellular conductivity was not determined, CSD waveforms cannot be considered absolute quantitative estimates of current density. Units were therefore not provided on CSD profiles. Data were analyzed using Clampfit 10.2 and GraphPad Prism 5.0. Values were given as mean \pm 
A
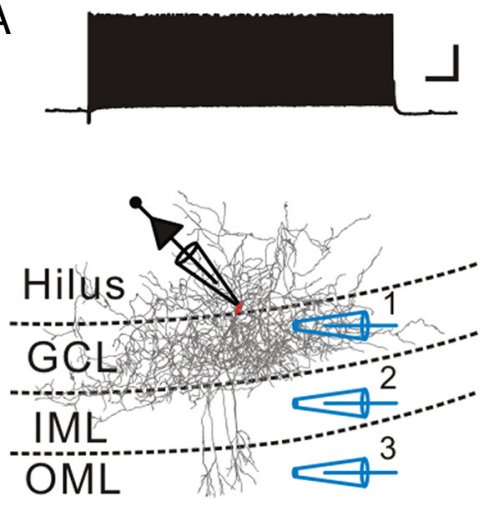

C

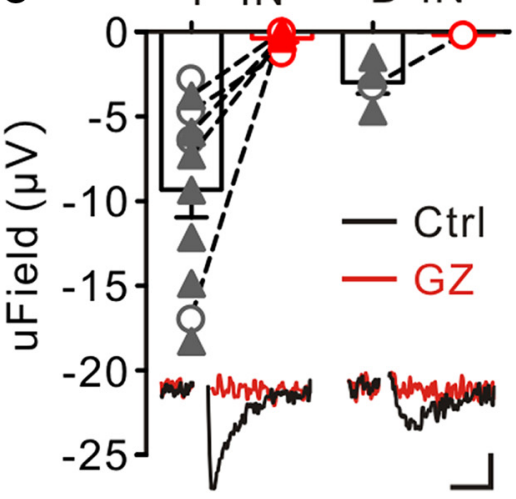

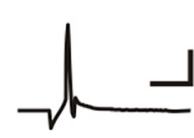

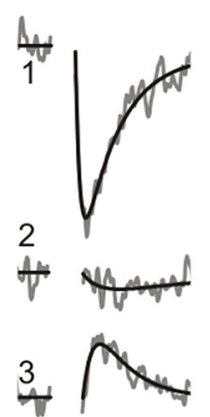

B
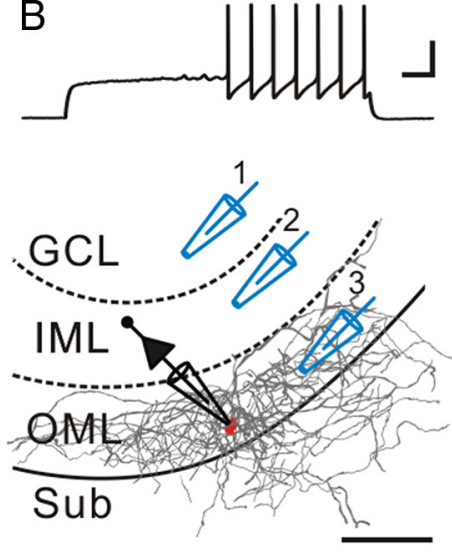
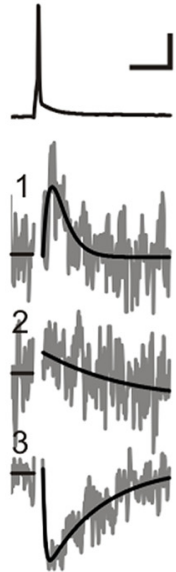

D

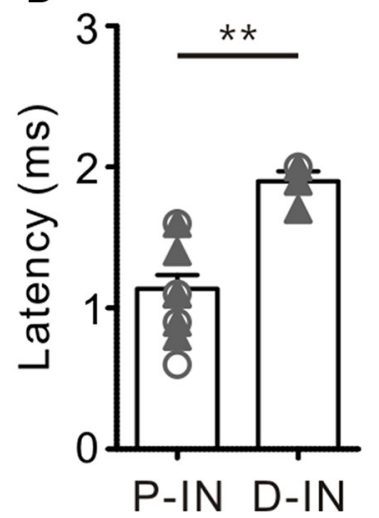

$\mathrm{E}$

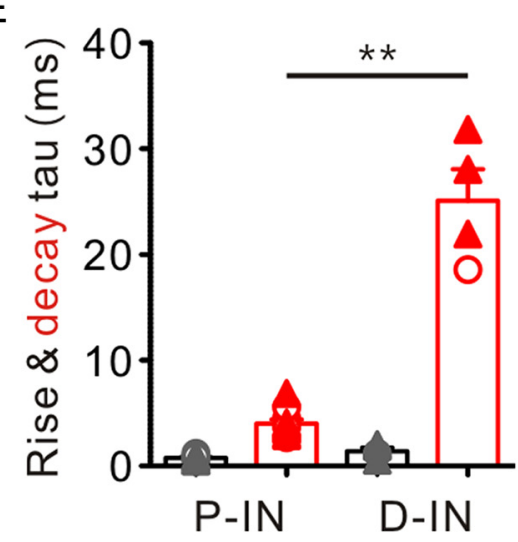

Figure 2. Extracellular uFields generated by P- or D-INs in the DG. A, Left, Experimental configuration, discharge pattern ( $+900 \mathrm{pA}$ current injection), and reconstruction of a biocytin-filled P-IN, whose axon arborized in the GCL. Calibration: $200 \mathrm{~ms}, 30 \mathrm{mV}, 100 \mu \mathrm{m}$. Right, uFields generated by evoking single APs in the same cell were recorded from the indicated regions. Calibration: $10 \mathrm{~ms}$, $30 \mathrm{mV}, 1.5 \mu \mathrm{V}$. B, Left, Experimental configuration, discharge pattern (+210 pA current injection), and a reconstructed D-IN. Note the axonal collaterals in the 0ML and subiculum (Sub). Calibration: $200 \mathrm{~ms}, 35 \mathrm{mV}, 100 \mu \mathrm{m}$. Right, uFields generated by evoking single APs in the same cell were recorded from the indicated regions. Calibration: $15 \mathrm{~ms}, 35 \mathrm{mV}, 1 \mu \mathrm{V}$. C, Summary of uFields evoked by P-INs and D-INs before and after GZ addition and their representative traces recorded from the GCL and $0 M L$, respectively. Calibration: $10 \mathrm{~ms}, 0.75 \mu$ V. Filled symbols in $\boldsymbol{C}-\boldsymbol{E}$ represent neurons having morphological reconstructions. $\boldsymbol{D}$, Summary of uField latency. ${ }^{* *} p<0.01$. $E$, Summary of $\tau_{\text {rise }}$ and $\tau_{\text {decay }}$ of uFields. ${ }^{* *} p<0.01$.

SEM. Statistical significance was tested by the nonparametric Wilcoxon signed-rank or ranked-sum tests.

Simulations. Simulations were performed using the NEURON 7.1 simulator (Hines and Carnevale, 1997). The DGC model (cell \#4 from Schmidt-Hieber et al., 2007) had homogeneously distributed parameters, including a specific intracellular resistivity $\left(R_{\mathrm{i}}\right)$ of $172.1 \Omega \mathrm{cm}$, a specific membrane capacitance $\left(C_{\mathrm{m}}\right)$ of $0.99 \mu \mathrm{F} / \mathrm{cm}^{2}$, and a specific membrane resistance $\left(R_{\mathrm{m}}\right)$ of $22.5 \mathrm{k} \Omega \mathrm{cm}^{2}$. In the active model, fast sodium and various types of potassium channels were distributed over soma, dendrites and axon as described previously (Aradi and Holmes, 1999). AP threshold was adjusted to $-40 \mathrm{mV}$. Voltage-gated channels were not included in the passive model. EPSPs $\left(1.0-40 \mathrm{nS} ; E_{\mathrm{AMPA}}=0\right.$ $\mathrm{mV})$ were generated by a conductance change with exponential rise $\left(\tau_{\text {rise }}=\right.$ $0.2 \mathrm{~ms})$ and decay $\left(\tau_{\text {decay }}=2.5 \mathrm{~ms}\right)$. GABAergic PSPs (GPSPs; $E_{\mathrm{GABA}}=$ $-72 \mathrm{mV}$ ) were generated by a conductance with a triple exponential time course $\left(10.6-20 \mathrm{nS} ; \tau_{\text {rise }}=0.19 \mathrm{~ms} ; \tau_{\text {decay-fast }}=1.9 \mathrm{~ms} ; \tau_{\text {decay-slow }}=9.4\right.$ $\mathrm{ms}$; amplitude ratio $=38: 62$ ) as measured previously (Kraushaar and Jonas, 2000). The $V_{\text {rest }}$ was adjusted to $-90 \mathrm{mV}$ in the passive model and $-85 \mathrm{mV}$ in the active model. Simulations were performed at $34^{\circ} \mathrm{C}$.

\section{Results}

\section{Region-specific GABAergic actions in the hippocampus}

To examine the action of GABA in the hippocampus during postnatal development, we recorded cFields in multiple layers of the CA areas and the DG in brain slices from 3- to 12-week-old rats. Extracellular cField recorded in the stratum pyramidale (SP) of the CA1 area in response to local stimulation of the axons (largely from P-INs including axo-axonic cells and basket cells) in the SP in the presence of $2 \mathrm{~mm}$ kynurenic acid (an ionotropic glutamate receptor blocker) displayed positive (upward) responses (142 \pm $16 \mu \mathrm{V} ; n=11$; Fig. $1 A, C)$ and could be blocked by the $\mathrm{GABA}_{\mathrm{A}}$ receptor antagonist GZ $(1 \mu \mathrm{M} ; n=7$; Fig. $1 D)$. Furthermore, the kinetics $\left(\tau_{\text {rise }}, 2.0 \pm 0.3 \mathrm{~ms} ; \tau_{\text {decay }}, 12.3 \pm 0.6 \mathrm{~ms} ; n=11\right.$; Fig. $\left.1 E\right)$ and paired pulse ratio (PPR; at $20 \mathrm{~Hz}, 0.69 \pm 0.05 ; n=6$ ) of the cField were similar to those of unitary IPSCs (Bartos et al., 2002). The cFields reversed polarity in the stratum oriens, stratum radiatum, and stratum lacunosum-moleculare (Fig. $1 \mathrm{~A}$ ). To determine the location of major current sources, we performed current-source density (CSD) analysis (Mitzdorf, 1985). CSD analysis revealed that the major current "source" signals were located within the SP (Fig. $1 A$, asterisk), indicating that synaptic GABA release from P-INs hyperpolarized the cell bodies of PNs. Notably, similar CSD (data not shown) and cField profiles were found in the CA3 area (Fig. 1D,E).

In striking contrast, cFields in the GCL were negative (141 \pm $10 \mu \mathrm{V} ; n=15$; Fig. $1 B, C$ ) while stimulating the axons within the GCL and reversed polarity in the inner and outer molecular layers (IML and OML). The cFields were also blocked by GZ and showed kinetics (Fig. $1 D, E)$ and PPR $(20 \mathrm{~Hz}, 0.74 \pm 0.02$; $n=10)$ similar to those in the CA areas. Because the GCL is largely innervated by the P-INs' axons, the negative cFields recorded in the GCL, reflecting a locally generated inward synaptic 

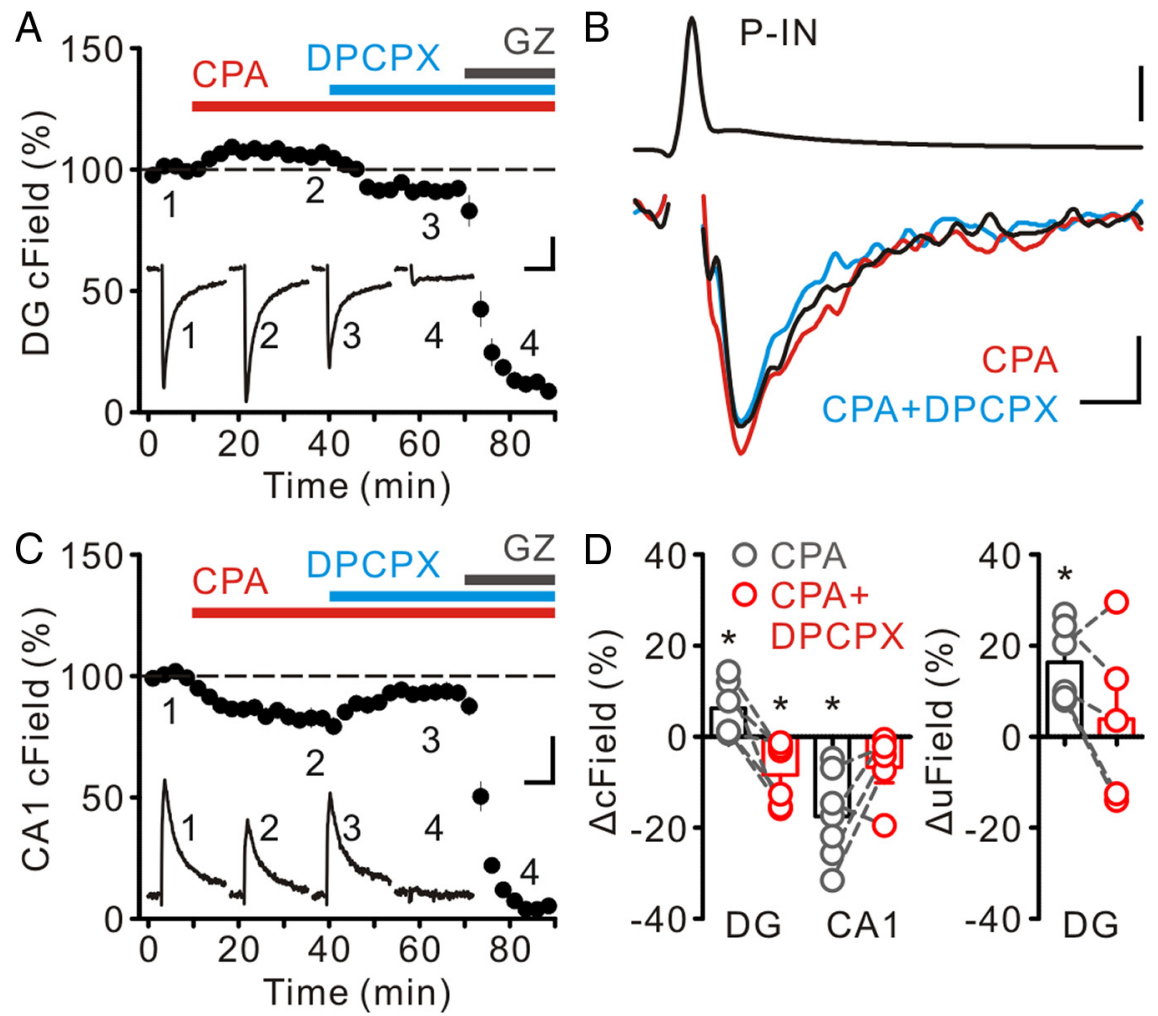

Figure 3. Differential modulation of cFields in the $D G$ and $C A 1$ via changes of $V_{\text {rest }}$. A, The GZ-sensitive cFields were recorded in the DG-GCL in response to extracellular stimulation of axons in the same layer. Bath-applied CPA increased the cFields, whereas further addition of DPCPX reduced the cFields. Calibration: $40 \mathrm{~ms}, 50 \mu \mathrm{V}$. B, The uFields evoked by activation of a P-IN in the DG in control (black), with CPA (1 $\mu \mathrm{m}$, red), or with CPA + DPCPX (5 $\mu \mathrm{m}$, blue). Calibration: $3 \mathrm{~ms}, 40 \mathrm{mV}, 3 \mu \mathrm{V}$. C, The cFields recorded in the CA1-SP area in response to extracellular stimulation of axons in the same layer. Bath-applied CPA reduced the CFields, whereas further addition of DPCPX reversed the effect. Calibration: $40 \mathrm{~ms}, 50 \mu$ V. D, Summary of the effects of CPA and DPCPX on the cFields and uFields recorded in the DG-GCL and CA1-SP areas, respectively. ${ }^{*} p<0.05$.

current. Consistent with this, CSD analysis revealed that the major current "sink" was located within the GCL (Fig. $1 B$, asterisk). These findings suggested that GABA depolarizes DGCs; a scenario remarkably different from that found in the CA areas (this study; Glickfeld et al., 2009; Bazelot et al., 2010).

To determine whether activation of single P-INs led to somatic depolarization of the DGCs, we recorded uFields in the GCL while eliciting APs in single fast-spiking $(>180 \mathrm{~Hz})$ interneurons in whole-cell patch recording at $34^{\circ} \mathrm{C}$. P-INs ( 7 of 11 cells) were identified by their extensive axonal arbors in the GCL. The uField $(9.3 \pm 1.6 \mu \mathrm{V} ; n=11$; Fig. $2 A)$ was negative and could be blocked by GZ ( $1 \mu \mathrm{M}$; Fig. $2 C$ ), suggesting that GABA-mediated inputs to the soma are depolarizing and lead to efflux of $\mathrm{Cl}^{-}$, resulting in a somatic current sink. Furthermore, the latency $(1.14 \pm 0.10 \mathrm{~ms} ; n=11$; Fig. $2 D)$ and kinetics $\left(\tau_{\text {rise }}, 0.75 \pm 0.07\right.$ $\mathrm{ms} ; \tau_{\text {decay }}, 4.02 \pm 0.37 \mathrm{~ms} ; n=11$; Fig. $2 E$ ) of uFields were similar to those generated by P-INs in the CA1-CA3 area (Glickfeld et al., 2009; Bazelot et al., 2010). When the recording pipette was moved to the IML, the uField was markedly decreased and reversed in polarity when the recording pipette was placed in the OML (Fig. 2A).

We next examined whether GABA release from D-INs led to dendritic depolarization of DGCs. We recorded uFields in the OML while evoking single APs in interneurons located in the OML ( 3 of 4 cells were identified post hoc as D-INs by their axonal arbors restricted in the OML; Fig. 2 B). All recorded interneurons in the OML displayed a late firing pattern, a ramp in membrane potential before APs (Fig. $2 B$, top trace), and no sag in response

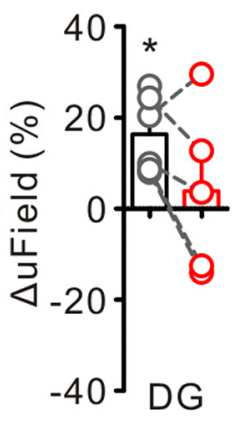

to a small hyperpolarizing current injection. These electrophysiological properties were similar to those of neurogliaform cells, a classical D-IN in the DG (Armstrong et al., 2011). Single APs induced in these cells evoked negative uFields (3.0 \pm $0.7 \mu \mathrm{V} ; n=4)$ in the OML, but positive uFields in the GCL (Fig. 2B). The latency $(1.90 \pm 0.07 ; n=4$; Fig. $2 D)$ and kinetics $\left(\tau_{\text {rise }}, 1.4 \pm 0.34 \mathrm{~ms} ; \tau_{\text {decay }}, 25.09 \pm 2.98\right.$ $\mathrm{ms} ; n=4$; Fig. $2 E$ ) of uField generated by D-INs were slower than those of P-INs.

\section{Mechanisms of depolarizing GABAergic responses}

To determine the sign of GABAergic transmission, we changed the driving force of GABAergic conductance via changing $V_{\text {rest }}$. Changes of $V_{\text {rest }}$ can result in either an increase or a decrease of cField, depending on where $E_{\mathrm{GABA}}$ lies with respect to the $V_{\text {rest }}$. We used the adenosine $A_{1}$ receptor agonist CPA to open potassium conductances (thereby hyperpolarize $V_{\text {rest }}$ ) and reversed its effect by the antagonist DPCPX. As previously reported (Glickfeld et al., 2009), we found that CPA and DPCPX had no effect on GABAergic transmission (data not shown). If the $E_{\mathrm{GABA}}$ is positive to the $V_{\text {rest }}$, then hyperpolarization of the DGCs through CPA will increase the driving force and thus increase the cField amplitude. Consistent with this idea, bathapplied CPA $(1 \mu \mathrm{M})$ enhanced the cField $(6.2 \pm 2.3 \%$ increase; $n=6 ; p<0.05$; Fig. $3 A)$ and uField $(16.4 \pm$ $3.2 \%$ increase; $n=6 ; p<0.05$; Fig. $3 B$ ) amplitudes; an effect that was reversed by $5 \mu \mathrm{M}$ DPCPX (uField, to $104 \pm 7 \%$ of control; $n=5 ; p=1.0$ compared with control; Fig. $3 B, D)$. Conversely, CPA resulted in a decrease $(17.5 \pm 3.4 \% ; n=7 ; p<0.05)$ of cField amplitude in the CA1 area, which was reversed by further addition of DPCPX (Fig. 3C,D).

\section{Modeling of GABAergic actions}

To better understand the impact of GABAergic input on synaptic integration in DGCs, we modeled GPSP-EPSP interactions in a multicompartment model of a DGC. We first examined the subthreshold interaction of somatic GPSPs with dendritic EPSPs in a passive model (Fig. 4A). When somatic GPSPs preceded $(-\Delta t)$ dendritic EPSPs, GPSPs passively propagated to the dendrite and enhanced the peak dendritic response (Fig. $4 B$, left, $C$, filled circles). Under this circumstance, this enhancement was dependent on GPSP timing, and only occurred when the GPSP was evoked before the dendritic EPSP (Fig. $4 C$, filled circles).

Next, we investigated the influence of GPSPs on AP generation in an active model (Fig. 4D). A near-threshold EPSP (red) was generated at a proximal dendrite (red dot). As exemplified in Figure $4 E$, both somatic and dendritic GPSPs facilitated AP generation (i.e., excitatory) when they preceded EPSPs by $30 \mathrm{~ms}$ (Fig. $4 E$, left). However, only dendritic but not somatic GPSPs facilitated AP generation when they preceded EPSPs by $10 \mathrm{~ms}$ (Fig. $4 E$, middle) and both of them had no facilitatory effect on AP initiation when coincident with EPSPs ( $\Delta t=0 \mathrm{~ms}$; Fig. $4 E$, right). By 
$\mathrm{D}$

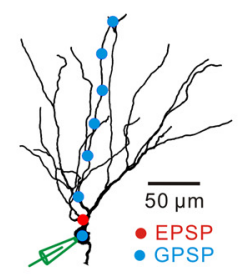

A

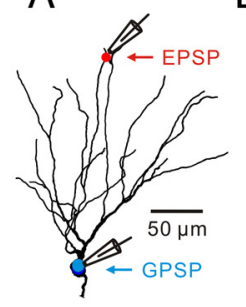

B Dendritic recording $(200 \mu \mathrm{m})$

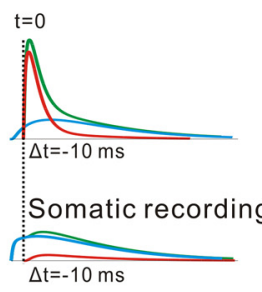

$t=0 \quad-E P S P+G P S P$

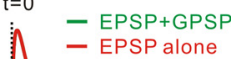

- EPSP+GPS
- EPSP alone

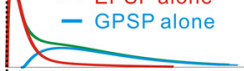

C

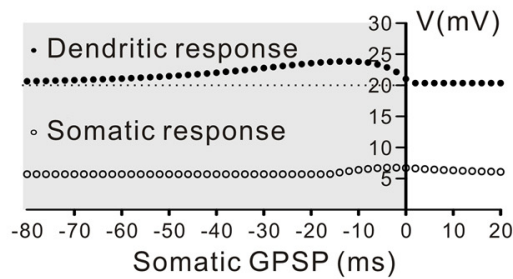

Somatic GPSP (ms)

$\mathrm{F}$
E Dendritic GPSP $(206 \mu \mathrm{m})$

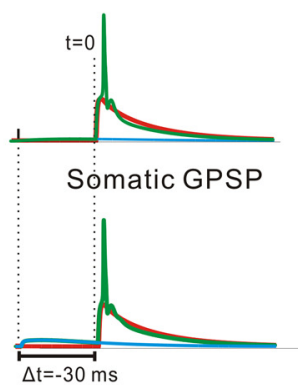

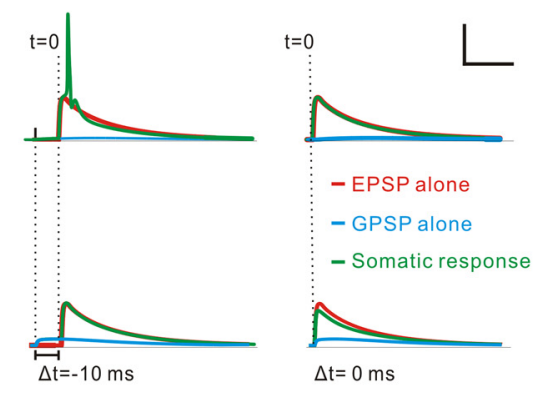

G Dendritic GPSP $(206 \mu \mathrm{m}) \mathrm{H}$

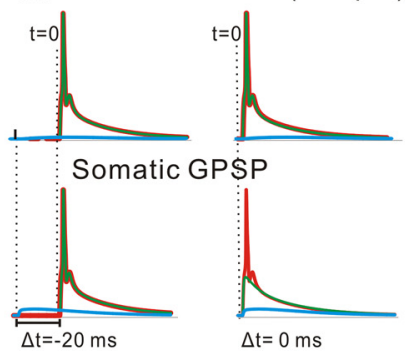

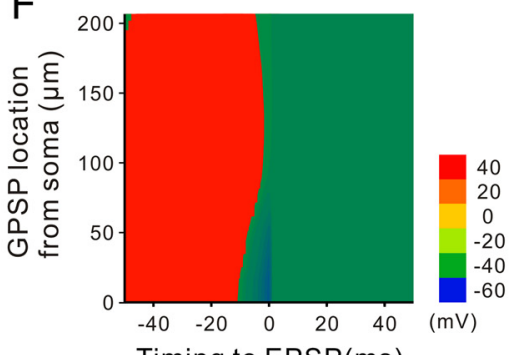

Timing to $\operatorname{EPSP}(\mathrm{ms})$

$\mathrm{t}=$

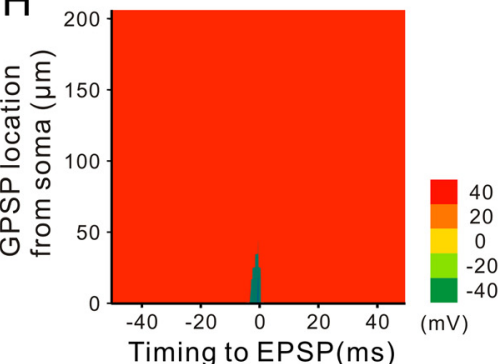

Figure 4. Synaptic interaction of GABAergic inputs with glutamatergic inputs. $\boldsymbol{A}$, Schematic diagram of a DGC model with a glutamatergic input (red) and a GABAergic input (blue). $\boldsymbol{B}$, Top left, Somatic GPSP preceded dendritic EPSP by $10 \mathrm{~ms}(\Delta t=-10 \mathrm{~ms})$; top right, somatic GPSP (blue) occurred $10 \mathrm{~ms}$ after $(\Delta t=+10 \mathrm{~ms})$ dendritic EPSP (red). GPSPs, EPSPs, and summated responses (green) were recorded at the dendritic site; Bottom, Similar to top, but responses were recorded from soma. Calibration: $20 \mathrm{~ms}, 10 \mathrm{mV}$. C, A plot of temporal summation of somatic GPSP and distal EPSP. Somatic GPSPs that occurred before $(-\Delta t)$ rather than after $(+\Delta t)$ dendritic EPSPs greatly enhanced dendritic responses. $D$, Schematic diagram of a DGC model with a proximal excitatory input (red) and single GPSPs (blue) across the somato-dendritic axis. $E$, Example of the timing of dendritic (top) and somatic (bottom) GPSPs relative to EPSPs determined the action of GABA and AP generation. Calibration: $20 \mathrm{~ms}, 40 \mathrm{mv} \boldsymbol{F}$, Plot of peak membrane potential (color code) against the GPSP timing and location relative to the subthreshold EPSP generated at the proximal dendrite. Note that both somatic and dendritic GABA conductances can facilitate AP generation (red shade) when timed to precede EPSP. G, Example of somatic GPSPs but not dendritic GPSPs suppressed AP initiation when coincident with a suprathreshold EPSP. $\boldsymbol{H}$, Plot of peak membrane potential (color code) against the GPSP timing and location relative to AP generated at the proximal excitatory synapse. Note that somatic and proximal $(<50 \mu \mathrm{m})$ GPSPs exerted shunting inhibition (green shade) near the occurrence of APs.

systematically examining the GPSP timing and location relative to the EPSP, we found that GPSPs, depending on their precise timing and location, can facilitate AP initiation when paired with the EPSP (Fig. 4F). Finally, to determine whether GABA can exert shunting inhibition during AP generation, a suprathreshold EPSP was generated at a proximal dendrite, which was large enough to evoke an AP (Fig. 4G, red). When somatic GPSPs occurred near the onset of EPSPs (e.g., $\Delta t=0 \mathrm{~ms}$ ), they suppressed AP generation (Fig. 4G, bottom). In contrast, dendritic GPSPs (>50 $\mu \mathrm{m}$ from soma) had no inhibitory effect on AP generation regardless of timing (Fig. $4 G$, top, $H$ ).

\section{Discussion}

GABAergic responses in rat hippocampal PNs are typically depolarizing during the first postnatal week and are transformed into being hyperpolarizing during later postnatal development. Contrary to this rule, we demonstrate that GABAergic signaling in the adult DG remains depolarizing. Analysis of DGC cable models reveals that GABAergic action, depending on its precise timing and location, could have an excitatory or inhibitory role in synaptic integration.

\section{Determining GABAergic actions using a noninvasive approach}

The polarity of GABAergic response is primarily governed by the relation of $V_{\text {rest }}$ and $E_{\mathrm{GABA}}$. However, measurements of $V_{\text {rest }}$ and $E_{\mathrm{GABA}}$ appeared to be nontrivial (see Glickfeld et al., 2009). Recent studies (Ge et al., 2006; Sun et al., 2009) have reported that GABAergic action switches from depolarizing to hyperpolarizing during maturation of DGCs. In their studies, $V_{\text {rest }}$ of DGCs remains at $\sim-60 \mathrm{mV}$ throughout all developmental stages, whereas $E_{\mathrm{GABA}}$ shifts from $\sim-40$ to $\sim-70 \mathrm{mV}$ during development. In contrast, several other groups have found that DGCs have very negative $V_{\text {rest }}$ (from -80 to $-85 \mathrm{mV}$ ) both in vitro (Staley and Mody, 1992; Schmidt-Hieber et al., 2004, 2007; Alle and Geiger, 2006; Krueppel et al., 2011) and in vivo (Penttonen et al., 1997).

To determine the nature of GABAergic transmission in the CA areas, Glickfeld et al. (2009) and Bazelot et al. (2010) elegantly avoided measuring $V_{\text {rest }}$ and $E_{\mathrm{GABA}}$ by combining uField recordings with post hoc identification of the location of activated GABAergic axons. Their approach, which we used here, has at 
least two advantages. First, it does not perturb $V_{\text {rest }}$ or intracellular ion concentrations. Second, it reports the average response of the cell population, and so is insensitive to variations across individual neurons. One disadvantage of this method, however, is that it would not be able to detect a small minority of DGCs whose response to $\mathrm{GABA}_{\mathrm{A}}$ receptor activation was hyperpolarizing. Similarly, it fails to selectively detect responses from mature DGCs. Thus, Fields may be potentially confounded by the responses of immature DGCs, which have very depolarized $E_{\mathrm{GABA}}$. However, the number of immature DGCs generated each month during adult neurogenesis is $<6 \%$ of the total size of the DGC population (Cameron and McKay, 2001), suggesting that newly generated DGCs may not have much contribution to the Field, an average response of the DGC population.

\section{Biphasic effect of GABA}

Consistent with most reports (see above discussion), we find that $V_{\text {rest }}$ of DGCs is $-88.3 \pm 1.1 \mathrm{mV}(n=14)$, more negative than $E_{\mathrm{GABA}}(-72 \mathrm{mV}$, Kraushaar and Jonas, $2000 ;-80.1 \pm 3.1 \mathrm{mV}$ in our experiments, $n=6)$. Notably, the $E_{\mathrm{GABA}}$ is below the AP threshold of DGCs (Schmidt-Hieber et al., 2004; Krueppel et al., 2011). Thus, $\mathrm{GABA}_{\mathrm{A}}$ conductance in mature DGCs will remain "shunting" when it occurs during AP generation. Consistently, analysis of the DGC model reveals that synaptic $\mathrm{GABA}_{\mathrm{A}}$ conductance can exert an inhibitory effect on AP generation (Fig. 4G,H). A previous report (Gulledge and Stuart, 2003), however, demonstrated that excitatory actions of GABA even though the $E_{\mathrm{GABA}}$ is below AP threshold can arise when GABAergic inputs are "temporally" or "spatially" isolated from concurrent excitation. Consistent with their findings, our simulation data indicate that GABAergic action, depending on its precise timing and location to other excitatory inputs, can also facilitate AP generation in DGCs (Fig. $4 E, F$ ). This observation prompts further investigation of the spatiotemporal interaction between GABAergic and other excitatory synaptic inputs in DGC dendrites.

In addition to synaptic GABA, ambient GABA may also influence DGC excitability via activation of extrasynaptic $\mathrm{GABA}_{\mathrm{A}}$ receptors. A recent study (Song et al., 2011) showed that tonic $\mathrm{GABA}_{\mathrm{A}}$ receptor conductance in hippocampal interneurons is depolarizing from rest, making baseline tonic $\mathrm{GABA}_{\mathrm{A}}$ conductance excitatory. However, increasing the tonic conductance can enhance shunting-mediated inhibition, which eventually overpowers the excitation. Consistent with their findings, we also find a biphasic effect of tonic GABA conductance on DGC firing; that is, low concentration $(1 \mu \mathrm{M})$ of bath-applied GABA increases glutamateinduced firing of DGCs, whereas high concentration $(100 \mu \mathrm{M})$ of GABA significantly depresses it (data not shown).

\section{References}

Alle H, Geiger JR (2006) Combined analog and action potential coding in hippocampal mossy fibers. Science 311:1290-1293.

Amaral DG, Scharfman HE, Lavenex P (2007) The dentate gyrus: fundamental neuroanatomical organization (dentate gyrus for dummies). Prog Brain Res 163:3-22.

Aradi I, Holmes WR (1999) Role of multiple calcium and calciumdependent conductances in regulation of hippocampal dentate granule cell excitability. J Comput Neurosci 6:215-235.

Armstrong C, Szabadics J, Tamás G, Soltesz I (2011) Neurogliaform cells in the molecular layer of the dentate gyrus as feed-forward GABAergic modulators of entorhinal-hippocampal interplay. J Comp Neurol 519:1476-1491.

Banke TG, McBain CJ (2006) GABAergic input onto CA3 hippocampal interneurons remains shunting throughout development. J Neurosci 26:11720-11725.

Bartos M, Vida I, Frotscher M, Meyer A, Monyer H, Geiger JR, Jonas P (2002) Fast synaptic inhibition promotes synchronized gamma oscillations in hippocampal interneuron networks. Proc Natl Acad Sci U S A 99:13222-13227.

Bartos M, Vida I, Jonas P (2007) Synaptic mechanisms of synchronized gamma oscillations in inhibitory interneuron networks. Nat Rev Neurosci 8:45-56.

Bazelot M, Dinocourt C, Cohen I, Miles R (2010) Unitary inhibitory field potentials in the CA3 region of rat hippocampus. J Physiol 588:2077-2090.

Ben-Ari Y (2002) Excitatory actions of GABA during development: the nature of the nurture. Nat Rev Neurosci 3:728-739.

Cameron HA, McKay RD (2001) Adult neurogenesis produces a large pool of new granule cells in the dentate gyrus. J Comp Neurol 435:406-417.

Ge S, Goh EL, Sailor KA, Kitabatake Y, Ming GL, Song H (2006) GABA regulates synaptic integration of newly generated neurons in the adult brain. Nature 439:589-593.

Glickfeld LL, Roberts JD, Somogyi P, Scanziani M (2009) Interneurons hyperpolarize pyramidal cells along their entire somatodendritic axis. Nat Neurosci 12:21-23.

Gulledge AT, Stuart GJ (2003) Excitatory actions of GABA in the cortex. Neuron 37:299-309.

Hines ML, Carnevale NT (1997) The NEURON simulation environment. Neural Comput 9:1179-1209.

Kraushaar U, Jonas P (2000) Efficacy and stability of quantal GABA release at a hippocampal interneuron-principal neuron synapse. J Neurosci 20:5594-5607.

Krueppel R, Remy S, Beck H (2011) Dendritic integration in hippocampal dentate granule cells. Neuron 71:512-528.

Ming GL, Song H (2011) Adult neurogenesis in the mammalian brain: significant answers and significant questions. Neuron 70:687-702.

Mitzdorf U (1985) Current source-density method and application in cat cerebral cortex: investigation of evoked potentials and EEG phenomena. Physiol Rev 65:37-100.

Penttonen M, Kamondi A, Sik A, Acsády L, Buzsáki G (1997) Feed-forward and feed-back activation of the dentate gyrus in vivo during dentate spikes and sharp wave bursts. Hippocampus 7:437-450.

Rivera C, Voipio J, Payne JA, Ruusuvuori E, Lahtinen H, Lamsa K, Pirvola U, Saarma M, Kaila K (1999) The $\mathrm{K}^{+} / \mathrm{Cl}^{-}$transporter KCC2 renders GABA hyperpolarizing during neuronal maturation. Nature 397:251-255.

Rivera C, Voipio J, Kaila K (2005) Two developmental switches in GABAergic signalling: the $\mathrm{K}^{+}-\mathrm{Cl}^{-}$cotransporter $\mathrm{KCC} 2$ and carbonic anhydrase CAVII. J Physiol 562:27-36.

Schmidt-Hieber C, Jonas P, Bischofberger J (2004) Enhanced synaptic plasticity in newly generated granule cells of the adult hippocampus. Nature 429:184-187.

Schmidt-Hieber C, Jonas P, Bischofberger J (2007) Subthreshold dendritic signal processing and coincidence detection in dentate gyrus granule cells. J Neurosci 27:8430-8441.

Song I, Savtchenko L, Semyanov A (2011) Tonic excitation or inhibition is set by $\mathrm{GABA}_{\mathrm{A}}$ conductance in hippocampal interneurons. Nat Commun 2:376.

Staley KJ, Mody I (1992) Shunting of excitatory input to dentate gyrus granule cells by a depolarizing $\mathrm{GABA}_{\mathrm{A}}$ receptor-mediated postsynaptic conductance. J Neurophysiol 68:197-212.

Sun B, Halabisky B, Zhou Y, Palop JJ, Yu G, Mucke L, Gan L (2009) Imbalance between GABAergic and glutamatergic transmission impairs adult neurogenesis in an animal model of Alzheimer's disease. Cell Stem Cell 5:624-633.

Weng JY, Lin YC, Lien CC (2010) Cell type-specific expression of acidsensing ion channels in hippocampal interneurons. J Neurosci 30:65486558 . 